\begin{tabular}{ccc}
\hline & International Journal of Engineering \& Technology, $7(2.7)(2018) 237-242$ \\
SPC & International Journal of Engineering \& Technology \\
Website: $w w w . s c i e n c e p u b c o . c o m / i n d e x . p h p / I J E T$ & Research Paper \\
\hline
\end{tabular}

\title{
Application of newton Raphson and steepest descent method for precise positioning for mobile communications
}

\author{
Ch. Yamini Surya Teja ${ }^{1 *}$, B. Vijay Gopal Reddy ${ }^{1}$, S. Sri Harsha ${ }^{1}$, K. Uday Kiran ${ }^{1}$, Dr. S. Koteswara Rao ${ }^{1}$ \\ ${ }^{1}$ Department of Electronics and Communication Engineering Koneru Lakshmaiah Education Foundation, \\ Vaddeswaram, Guntur, Andhra Pradesh, India - 522502 \\ *Corresponding author E-mail: c.yamini11@gmail.com
}

\begin{abstract}
GSM is a wireless network system. GSM mostly uses TDMA and most uses three digital wireless mobile network technologies to compresses data, sends by using a channel along with other samples of data, in different time slots. It makes use of 900 to $1800 \mathrm{MHz}$ band frequency for transmission. Very accurate and exact wireless algorithms are difficult in all environmental conditions. Wireless communications use various parametric measurements to determine the sensor position. TOA is the time taken by the signal from source to reach the receiver's position. Newton Raphson method is an iterative numerical method uses partial derivatives of a functions or a system of equations in a suitable search direction. Newton Raphson consider two fundamental arguments: The first one is considering a good starting or initial point which is approximate to the solution and the secondary thing is to consider a distinguishable error which plays important role on approximation of the solution. In the present work, the Newton Raphson algorithm is implemented to obtain precise position of the receiver.
\end{abstract}

Keywords: Global System for Mobile Communication; TOA; Newton Raphson Method; Steepest Descent Method; Signal Processing Algorithms.

\section{Introduction}

Positioning algorithms determine the location of a person or an object either with respect to a known position or with respect to a coordinate system. It provides highly accurate results in the implementation of localization in a wireless sensor network (WSN), i.e. to estimate the position of a sensor node, termed as complex challenge in wireless networks. In the previous decades, multiple positioning systems have been introduced to develop highly-accurate, low-power, low-cost localization schemes due to degradation of real world applications, navigational systems, civil, military surveillance and emergency services. In case of any accident if we are late by few seconds it leads to the life threatening of injured victim. The requirement for accurate and optimal localization schemes have been even motivated by demand with the rapid development of cost-effective Micro-Electro-Mechanical System (MEMS). Furthermore, to broadcast the signals to longer distances in multiple paths with lower signal strength at the receiver, localization algorithms are used. Wireless networks take the help of radio waves and/or microwave to take care the communication channels in between the sensor nodes.

Each sensor in the mobile network is equipped with sensing the signal, computation for the required results and obtaining the final results. During the last few decades, different methods are implemented to define the accurate position of the sensor node. The strength of the signal received at the three or more sensor nodes determines the unknown position of the source (i.e.)., trilateration can be achieved.

\subsection{Arrival angle}

Arrival Angle is important method used to determine the source position. Time-difference-of-arrival and time-of-arrival are part of category of time based localization. Ironically, these methods take the time as reference moving from source to receiver to estimate the position. RSS and TDOA are more evolving in mobile communications due to the flexibility of implementation. In case of AOA, expensive antennas are required to estimate the position if we take the consideration of time based localization synchronization between receiver and the source is required.

To increase the performance of localization, we can use a hybrid localization method where two methods are combined, for example, the mixture of RSS and AOA was determined. Consider the receiver node is a small and costless device, which is a combination of RSS and TDOA can be considered. All these localization schemes have one limitation in common, which can be easily destroyed by the non line of sight situations or multipath fading.

\subsection{Source localization}

The localization of a source, i.e. identification of a source node's location in a mobile communication network, is a challenging problem. The knowledge of this work is to develop the accurate, cost effective and valid localization algorithms for localization in mobile communications. It describes the overall view of existing localization techniques, their limitations and its applications. NewtonRaphson method with the TOA, RSS, TDOA, RSS schemes along with their results. It also describes about the applications of proposed method. Steepest Descent method with the TOA, RSS, TDOA, RSS schemes along with their results. It also describes about the applications of proposed method. 
It provides conclusive remarks and feasible future direction of the research. Finally, the project ends with appendix which cover how to obtain the MATLAB source code of algorithm implementations (Appendix A). It provides highly accurate results in the implementation of localization in a wireless sensor network (WSN), i.e. to find the position of a sensor node, termed as complex challenge in wireless sensor networks.

\section{Source localization}

Localization method determines the position of a person or a thing within the stipulated co-ordinate system and to determine the accurate and exact position. It can also use the error distribution for exact positioning. During many years, several methods have been developed to provide an ex act location of an unknown sensor node.

\subsection{Categories of localization methods}

As shown in Figure 2.1, categories of localization algorithm for precise positioning:

Range Positioning:

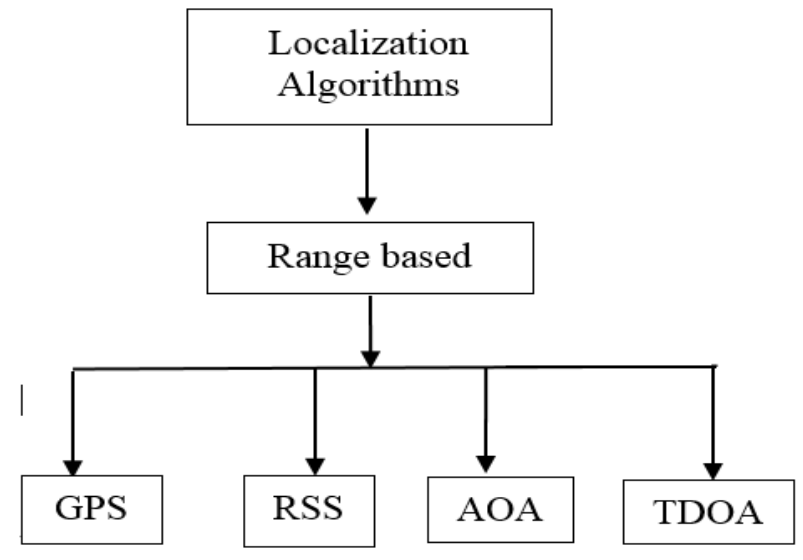

Fig. 2.1:

The classification is depended on the information used for source localization. Range-based methods used the information from range measurements such as direction and angle of measurement. Several range-based location technologies exist, with methods that vary from low accuracy based on cell identification to high accuracy, but costly approaches have been developed to address the sensor localization methods.

The Range-based techniques have used Received Signal Strength, Time of Arrival, Angle of Arrival and Time Difference of Arrival The rest of the chapter will discuss about the range based techniques and comparing the techniques with newly proposed techniques in terms of ease of implementation, better efficiency and accurate results of localization techniques. Mathematical methodologies that are utilized for solving the localization problem.

\subsection{Importance of wireless sensor networks localization}

They examined the problem of source localization in wireless networks using different parameters. They developed direct methods for obtaining the source position. They also considered white gaussian noise in determining the source position. The solutions obtained from the TOA are very useful when compared to that of the TDOA measurements. The proposed method is susceptible to the $3 \mathrm{db}$ noise measurements and signal transmission chracteristics.

\subsection{Newton-raphson method with noisy function meas- urements}

The improvement of the suggested ideal calculation is dependent upon minimizing a stochastic execution. Those estimation lapse covariance grid will be demonstrated to meet on zero to linearized capacities same time recognizing added substance zero-mean white noise. The paper recommended a novel recursive algorithm giving ideal iterative-varying addition to linearized works. It was analytically demonstrated that this proposed approach can provide zero convergence of the error covariance matrix for linear function in presence of measurement noise.

\section{Proposed methodology}

As we know, several methods are used for the source localization we are particularly concentrated on the TOA which is required to evaluate the problem of position estimation and in addition to know the time of emission. The nonlinear method designed to estimate the source location directly includes NLS and ML estimators. In NLS estimator the accuracy is generally high, and the noise statistics are not needed.

Localization problems based on triangulation consist of two methods: lateration and angulation. Lateration methods defines the location estimation by considering distances from multiple reference points instead angulation, measure angles relative to several source and receiver points. For example, lateration methods can be based on noisy received signal strength measurements. Other applications include design of composite materials recovery of sparse and compressible signals such as image sampling, color analysis and acoustic wave propagation in turbulent fluids. Optimization methods are frequently implemented in such noisy-based applications. However, stochastic optimization techniques provide an effective approach in the presence of noisy measurements.

While considering gradient based descent approach, numerous techniques have been proposed to enhance the decisions of the step size, for example, the ideal adaptation of Robbins-Monro (RM) calculation, a quickened RM calculation and a quickened variant of Kesten calculation. Stable constants are used to step size to increase the numerical ability. An efficient approach for achieving a second order adaptive algorithm is presented where two parallel recursions are implemented for estimating the solution using NR algorithm, and the other for estimating the Hessian matrix. Stochastic gradient algorithm for a calculation of the parameters while the others are held fixed is mentioned in an effective approach. The above method is not affected by the noise consideration considered.

\subsection{Newton raphson algorithm}

The Newton-Raphson method is most known numerical method for the outcome of NLS problems. This method requires calculation of gradient of objective function $\nabla \mathrm{J}_{\mathrm{NLS}}(\mathrm{x})$ and Hessian matrix $\nabla^{2} \mathrm{~J}_{\mathrm{NLS}}(\mathrm{x})$, the gradient is expressed as follows

$\nabla \mathrm{J}_{\mathrm{NLS}}(\mathrm{x})=\left[\begin{array}{l}\frac{\partial J_{N L S}(x)}{\partial x} \\ \frac{\partial J_{N L S}(x)}{\partial y}\end{array}\right] \in \mathrm{R}^{2}$,

$=-2\left[\begin{array}{c}\sum_{k=1}^{k} \frac{\left(r_{T O A}-\left(x-x_{1}\right) \sqrt{\left(x-x_{1}\right)^{2}+\left(y-y_{1}\right)^{2}}\right)}{\left[\left(x-x_{1}\right)^{2}+\left(y-y_{1}\right)^{2}\right]^{1 / 2}} \\ \sum_{k=1}^{k} \frac{r_{T O A}-\left(y-y_{1}\right) \sqrt{\left(x-x_{1}\right)^{2}+\left(y-y_{1}\right)^{2}}}{\left[\left(x-x_{1}\right)^{2}+\left(y-y_{1}\right)^{2}\right]^{1 / 2}}\end{array}\right]$

$\nabla^{2} \mathrm{~J}_{\mathrm{NLS}}(\mathrm{x})=\left[\begin{array}{ll}\frac{\partial^{2} J_{N L S}(x)}{\partial x^{2}} & \frac{\partial^{2} J_{N L S}(x)}{\partial x \partial y} \\ \frac{\partial^{2} J_{N L S}(x)}{\partial x \partial y} & \frac{\partial^{2} J_{N L S}(x)}{\partial y^{2}}\end{array}\right] \in R^{2 x 2}$

Where, 


$$
\begin{aligned}
& \frac{\partial^{2} J_{N L S}(x)}{\partial x \partial y}=\frac{\partial^{2} J_{N L S}(x)}{\partial y \partial x}=\sum_{K=1}^{K} \frac{2 r_{T O A}\left(x-x_{1}\right)\left(y-y_{1}\right)}{\left[\left(x-x_{1}\right)^{2}+\left(y-Y_{1}\right)^{2}\right]^{3 / 2}} \\
& \frac{\partial^{2} J_{N L S}(x)}{\partial y^{2}}= \\
& \sum_{K=1}^{K} 2\left\lceil\frac{\left(y-y_{1}\right)^{2}}{\left(x-x_{1}\right)^{2}+\left(y-y_{1}\right)^{2}}-\frac{\left(r_{T O A}-\sqrt{\left(X-X_{1}\right)^{2}+\left(Y-Y_{1}\right)^{2}}\right)\left(X-X_{1}\right)^{2}}{\left[\left(x-x_{1}\right)^{2}+\left(y-y_{1}\right)^{2}\right]^{3 / 2}}\right\rceil \\
& \frac{\partial^{2} J_{N L S}(x)}{\partial x^{2}}= \\
& \sum_{K=1}^{K} 2\left\lceil\frac{\left(x-x_{1}\right)^{2}}{\left(x-x_{1}\right)^{2}-\left(y-y_{1}\right)^{2}}-\frac{\left(r_{T O A}-\sqrt{\left(x-x_{1}\right)^{2}+\left(y-y_{1}\right)^{2}}\right)\left(y-y_{1}\right)^{2}}{\left[\left(x-x_{1}\right)^{2}+\left(y-y_{1}\right)^{2}\right]^{3 / 2}}\right\rceil
\end{aligned}
$$

The objective function $\mathrm{J}_{\mathrm{NLS}}(\mathrm{x}+\Delta \mathrm{x})$ is given by the expansion of Taylor series expansion of the iteration as follows:

$\mathrm{J}_{\mathrm{NLS}}(\mathrm{x}+\Delta \mathrm{x}) \approx \varphi(x)$

Where

$\varphi(x)=J_{N L S}(x)+\Delta x^{T}\left[\nabla J_{N L S}(x)\right]+\frac{1}{2} \Delta x^{T} \nabla^{2} J_{N L S}(x) \Delta x$,

Where $\Delta x$ is a small change $\mathrm{x} \in \mathrm{R}^{2}$. The conditions for the function $\varphi(x)$ is

$\nabla \varphi(x)=\nabla J_{N L S}(x)+\nabla^{2} J_{N L S}(x) \Delta(x)=0$.

The step size is obtained as follow

$\Delta x=-\left[\nabla^{2} J_{N L S}(x)\right]^{-1} \nabla J_{N L S}(x)$.

$x^{(T+1)}=x^{(T)}+\Delta x^{(T)}$.

Where $x^{T}$ is the Tth iteration. The iterative process i.e NewtonRaphson method is continued until the gradient of $\mathrm{J}_{\mathrm{NLS}}(\mathrm{x})$ follows the condition

$\left\|\nabla J_{N L S}\left(x^{(T+1)}\right)\right\| \leq \varepsilon$.

\subsubsection{Hybrid generic newton raphson implementation}

The algorithm depends on global as well as normal search to increase performance of normal Newton Raphson algorithm. The basic thought of implementing hybrid algorithm is to increase the advantages over normal algorithm by decreasing the disadvantages of normal one and to increase the efficiency. The NR method is efficient method, but its convergence is less when compared to the starting point. Hence, the search algorithm, is applicable to choosing starting point in the search localization problem for the NR algorithm without trapping, and to achieve a faster output rate. The proposed hybrid GA-NR method for the NLS localization problem is then applied to estimate the position of the source in the LOS environments.

\subsection{Steepest descent algorithm}

Steepest Descent Method is one of the very popular methods of Gradient-based descent methods. When we apply the gradientbased descent methods, it requires the continuous partial derivatives. The partial functions are used to correct the weights after each pattern or summer to obtain the overall gradient. Let $g_{K}$ as the gradient in error function that is $\left[g_{K}=\nabla E\left(w_{k}\right)\right]$. The basic idea of the proposed method is that can reduce many sub-function in every iteration. By removing the several largest sub-functions, a descent direction can be obtained.

$w_{K+1}=w_{k}-\epsilon g_{k}$

Where,

$$
w_{k+1}=w_{k}-\epsilon \nabla E_{p}\left(w_{K}\right)
$$

Where $\epsilon>0$ is a step size of the gradient. After multiple iterations of algorithm on the surface, with $E_{p}\left(w_{0}\right) \geq E_{p}\left(w_{1}\right) \geq \cdots \geq$ $E_{p}\left(w_{k}\right)$ the sequence of $w_{k}$ converges to local minimum.

The value of the step size should vary at every iteration of the algorithm. Since the direction of gradient can be calculated as the first derivative of the function, the offset in the step size, requires extra pre-processing. Various formulation exists for the step size estimation, and here we make use of thee step size $\epsilon$.

To obtain the gradient $\nabla E$ for steepest descent algorithm, we take partial derivative of the error function $\mathrm{E}$ wrt $\mathrm{x}$ and $\mathrm{y}$ coordinates of source:

$\frac{\partial E}{\partial x}=2 c \frac{\Gamma_{A B}-\hat{\Gamma}_{A B}(w)}{\sigma_{A B}{ }^{2}}\left(\frac{x_{A}-x}{\sqrt{\left(x_{A}-x\right)^{2}+\left(y_{A}-y\right)^{2}}}-\frac{x_{B}-x}{\sqrt{\left(x_{B}-x\right)^{2}+\left(y_{B}-y\right)^{2}}}\right)$

$\frac{\partial E}{\partial y}=2 c \frac{\Gamma_{A B}-\hat{\Gamma}_{A B}(w)}{\sigma_{A B}{ }^{2}}\left(\frac{y_{A}-y}{\sqrt{\left(x_{A}-x\right)^{2}-\left(y_{A}-y\right)^{2}}}-\frac{y_{B}-y}{\sqrt{\left(x_{B}-x\right)^{2}-\left(y_{B}-y\right)^{2}}}\right)$

We can achieve the iterative solution for $w(x, y)$ using the steepest descent gradient method:

$x_{n+1}=x_{n}+\sum_{i=1}^{N} 2 \operatorname{c\varepsilon } \frac{\Gamma_{i}-\Gamma_{i}(w)}{\sigma_{i}^{2}}\left[\frac{x_{0}-x_{n}}{d_{w 0}}-\frac{x_{i}-x_{n}}{d_{w i}}\right]$

$y_{n+1}=y_{n}+\sum_{i=1}^{N} 2 c \varepsilon \frac{\Gamma_{i}-\Gamma_{i}(w)}{\sigma_{i}{ }^{2}}\left[\frac{y_{0}-y_{n}}{d_{w 0}}-\frac{y_{i}-y_{n}}{d_{w i}}\right]$

Where,

$d_{w 0}=\sqrt{\left(x_{0}-x_{n}\right)^{2}+\left(y_{0}-y_{n}\right)^{2}}$

And

$d_{w i}=\sqrt{\left(x_{i}-x_{n}\right)^{2}+\left(y_{i}-y_{n}\right)^{2}}$.

The linear stepped-frequency waveform is extremely used in radar and communication system, because they provide desirable performance improvement by exploring frequency diversity and are easily generated. The stepped-frequency waveform, by focusing an inverse fast Fourier transform (IFFT) to a bunch of narrowband pulses, achieves a wide bandwidth without use of excessive system complexity and cost. However, the generated pulse of a steppedfrequency waveform is sinc type; and it has a high first sidelobe, which needs to be suppressed by spectral weighting or mismatch method at the expense of main lobe width and signal-to-noise ratio. Moreover, due to the periodic response of IFFT at a digital frequency of $2 \pi$ radians per sample, stepped-frequency waveform will experience from large grating lobes when the step frequency is larger than the bandwidth of a single pulse. As a result, steppedfrequency waveform can only achieve a limited bandwidth given the number of pulses. Unlike the stepped-frequency waveform, hopped-frequency waveform uses irregularly or even randomly frequency hopping pattern.

As a result, periodic grating lobes are converted into sidelobes to achieve a wider bandwidth with limited constant pulses number becomes possible. Moreover, the incoherent diversity and flexibility in frequency domain grant hopped-frequency waveform several advantages, such as resistance to narrowband interference, low probability of intercept and sidelobe suppression potential.

For frequency waveform, an additional component is included to the sidelobes due to its irregularly distributed frequencies; and achieve a low sidelobe level poses a special frequency. This frequency can be delivered by applying a density tapered design. For example, proposed a nonlinear stepped-frequency waveform whose frequency density would approximate a window technique. These methods above are generally easy for implementation; however, 
their results are far from optimality in terms of sidelobe level and they cannot control the shape of the compressed pulse accurately either.

\subsection{Measurement of parameters used in both the meth- ods}

a) Received Signal Strength

In the absence of measurement errors and noise, the received signal strength from the unknown source measured at the ith receiver, can be modelled as

$P_{i}^{r}=k_{i} \frac{P_{i}^{t}}{d_{i}^{a}}, \mathrm{i}=1,2, \ldots, \mathrm{N}$

$P_{i}^{t}$ is the transmitted power sensor node, and di is the distance from source to the ith receiver. Furthermore, $\mathrm{Ki}$ accounts for all other factors at the received power, including antenna heights and gains, and $\mathrm{a}$ is the path loss constant. For free space $\mathrm{a}=2$. Without loss of generality, it is expected that $P_{i}^{t}, \mathrm{Ki}$, and are known beforehand. Then, considering measurement errors, the log-normal path loss model can be expressed as

$\ln \left(P_{i}^{r}\right)=\ln \left(K_{i}\right)+\ln \left(P_{i}^{t}\right)-\mathrm{a} \ln \left(d_{i}\right)+n_{r s s, i}, \mathrm{i}=1,2, \ldots, \mathrm{N}$

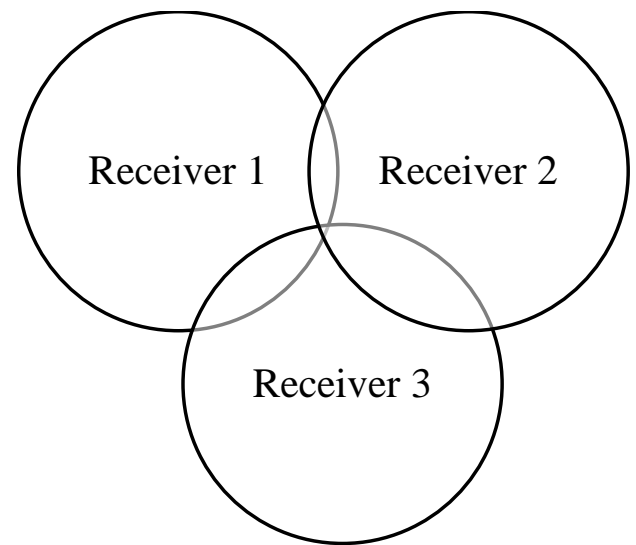

Fig.3.1: Intesection of Circles to Provide the Exact Position of Source.

$r_{r s s, i}=\ln \left(P_{i}^{r}\right)-\ln \left(K_{i}\right)-\ln \left(P_{i}^{t}\right)$

The RSS model can be given as

$r_{r s s, i}=-\mathrm{a} \ln \left(d_{i}\right)+n_{r s s, i}, \mathrm{i}=1,2, \ldots \ldots, \mathrm{N}$

The vector form of (3.4) is given as

$r_{r s s}=f_{r s s}(\mathrm{x})+n_{r s s}$

Where

$r_{r s s}=\left[\begin{array}{ll}r_{r s s, 1} & r_{r s s, 2 \ldots \ldots . . .} r_{r s s, N}\end{array}\right] \mathrm{T}$

$n_{r s s}=\left[\begin{array}{ll}n_{r s s, 1} & n_{r s s, 2 \ldots \ldots} \ldots n_{r s s, N}\end{array}\right]^{\mathrm{T}}$

And

$f_{r s s}(x)=-a\left[\begin{array}{c}\ln \left(\sqrt{\left(x-x_{1}\right)^{2}+\left(y-y_{1}\right)^{2}}\right) \\ \ln \left(\sqrt{\left(x-x_{1}\right)^{2}+\left(y-y_{1}\right)^{2}}\right) \\ \cdot \\ \cdot \\ \cdot \\ \ln \left(\sqrt{\left(x-x_{N}\right)^{2}+\left(y-y_{1}\right)^{2}}\right)\end{array}\right]$
The source localization process is to estimate the source position that is based on $r_{r S S}$ comes from the actual measurements. The above description describes estimators that can be used to solve equation (3.1.5). While RSS is simple to implement, as it avoids over usage of computers which creates problem in the outdoor locations.

b) Arrival time (TOA)

The time of arrival (TOA) is time taken by the signal to reach from source to destination. To obtain the TOA measurement at more than one receiver, it is required that the source and receivers precisely be synchronized. This can be destroyed by round or two-way trip. The centralized product of the TOA and speed of propagation denoted by $\mathrm{c}$ gives the distance between source and destination. In a 2-D plane and in the absence of measurement error, each TOA corresponds to a circle centered at a receiver. The intersection of three or more circles result in the source sensor location. In a 2-D localization setup, two TOA measurements will produce two circles and will have two possible estimations. These circles may not intersect at the same point in the presence of measurement errors and other disturbance. This leads the TOA problem into an optimization problem before the solution estimation. In the absence of measurement errors or disturbances, the TOA measured at the ith receiver, is denoted by $t_{i}$ and given by

$t_{i}=\frac{d_{i}}{c}, \mathrm{i}=1,2, \ldots, \mathrm{N}$

Where $\mathrm{c}$ is the speed of light

In the presence of disturbance, noise and measurement errors, the range measurement based on $\boldsymbol{t}_{\boldsymbol{i}}$ is given in terms of $\boldsymbol{r}_{\boldsymbol{t o a}, \boldsymbol{i}}$ and is denoted as

$r_{\text {toa }, i}=d_{i}+n_{\text {toa }, i}=\sqrt{\left(x-x_{1}\right)^{2}+\left(y-y_{1}\right)^{2}}+n_{\text {toa }, i}, i=$ $1,2, \ldots, N$

Where $n_{\text {toa }, i}$ is the range error in $r_{\text {toa }, i}$.

$n_{\text {toa }, i}$ is a Gaussian random variable with zero mean and TOA estimation variance. In vector form, equation (3.2.2) can be expressed as

$r_{\text {toa }}=f_{\text {toa }}(\mathrm{x})+n_{\text {toa }}$

Where

$r_{\text {toa }}=\left[\begin{array}{lll}r_{\text {toa }, 1} & r_{\text {toa }, 2} & \ldots \\ r_{\text {toa }, N}\end{array}\right]^{T}$

$n_{\text {toa }}=\left[n_{\text {toa }, 1} n_{\text {toa }, 2} \ldots n_{\text {toa }, N}\right]^{T}$

$f_{\text {toa }}(x)=\left[\begin{array}{c}\sqrt{\left(x-x_{1}\right)^{2}+\left(y-y_{1}\right)^{2}} \\ \sqrt{\left(x-x_{2}\right)^{2}+\left(y-y_{2}\right)^{2}} \\ \cdot \\ \cdot \\ \cdot \\ \sqrt{\left(x-x_{N}\right)^{2}+\left(y-y_{N}\right)^{2}}\end{array}\right]$

The requirement for synchronization between source and destination is TOA measurement. The source localization problem based on TOA measurements is to then estimate $\mathrm{x}$ given that $r_{\text {toa }}$ comes from the actual measurements.

c) Time Difference of Arrival (TDOA)

The time difference of arrival (TDOA) is the difference in TOAs of the received signal at a pair of receiver sensors, with respect to another receiver. Like TOA, it also requires synchronization between the source node and receiver rather than at the source position. The product of propagation speed leads to the range difference between the source node and the two receivers. In a noise free environment each TDOA produces a hyperbolic pattern on which the source may originates like in a 2-D plane. The target location is then produced by the intersection of two or more hyperbolic patterns. In the presence of disturbance and measurement errors, the source location is 
originated from a set of hyperbolic equations obtained from the TDOA measurements.

In the absence of measurement errors and disturbances, using 1st receiver as the reference, it can be easily shown that

r_(tdoa,i) $=\left(d \_i-d \_1\right)+$ n_(tdoa,i)

$=\sqrt{\left(x-x_{i}\right)^{2}+\left(y-y_{i}\right)^{2}}-\sqrt{\left(x-x_{1}\right)^{2}+\left(y-y_{1}\right)^{2}}+$

$n_{\text {tdoa }, i}, i=2, \ldots, N$

Where $n_{t d o a, i}$ is the error range in $r_{t d o a . i}$

If the TDOA measurements are directly obtained from TOA measurements, then it is given by

$n_{\text {tdoa }, i}=n_{\text {toa }, i}-n_{\text {toa }, 1}, \mathrm{i}=1,2, \ldots, \mathrm{N}$

In vector form it is given by,

$r_{\text {tdoa }}=f_{\text {tdoa }}(\mathrm{x})+n_{\text {tdoa }}$

Where

$$
\begin{aligned}
& r_{\text {tdoa }}=\left[\begin{array}{lll}
r_{\text {tdoa }, 2} & r_{\text {tdoa }, 3} \ldots r_{\text {tdoa }, N}
\end{array}\right]^{T} \\
& n_{\text {tdoa }}=\left[\begin{array}{lll}
n_{\text {tdoa }, 2} & n_{\text {tdoa }, 3} \ldots n_{t d o a, N}
\end{array}\right]^{T}
\end{aligned}
$$

$f_{\text {tdoa }}(x)=$

$\left[\begin{array}{c}\sqrt{\left(x-x_{2}\right)^{2}+\left(y-y_{2}\right)^{2}}-\sqrt{\left(x-x_{1}\right)^{2}+\left(y-y_{1}\right)^{2}} \\ \sqrt{\left(x-x_{3}\right)^{2}+\left(y-y_{3}\right)^{2}}-\sqrt{\left(x-x_{1}\right)^{2}+\left(y-y_{1}\right)^{2}} \\ \cdot \\ \cdot \\ \cdot \\ \sqrt{\left(x-x_{N}\right)^{2}+\left(y-y_{N}\right)^{2}}-\sqrt{\left(x-x_{1}\right)^{2}+\left(y-y_{1}\right)^{2}}\end{array}\right]$

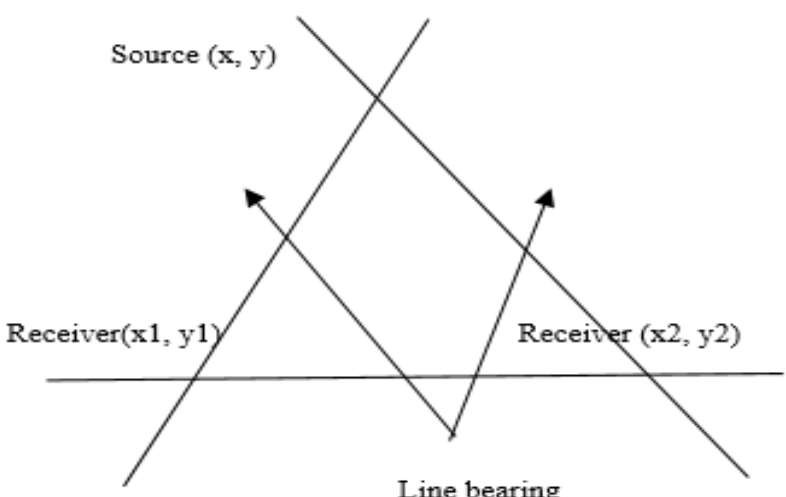

Fig. 3.2: Intersection of Two or More Lines to Provide the TDOA Estimation.

The source localization problem is to estimate location of position $\mathrm{x}$ based on $r_{\text {tdoa }}$. To facilitate the development and analysis of the localization scheme, $n_{t d o a, i}$ are assumed to be zero-mean and Gaussian distribution.

d) Angle of Arrival (AOA)

Time of arrival (AOA) is angle of arrival at the source when the signal reaches from source to the receiver. From each AOA, a line of bearing (LOB) can be assumed from source node to receiver node, and coincidence of two LOB provides possible location estimation from source to receiver. Although it does not require any synchronization between source and receiver, though it requires costly antenna arrays for location estimation using AOA measurement.

The AOA of the transmitted signal from the source at the ith receiver denoted by

$r_{a o a, i}=\alpha_{i}+n_{a o a, i}=\tan ^{-1}\left(\frac{y-y_{i}}{x-x_{i}}\right)+n_{a o a, i}, i=1,2, \ldots, N$
Where $n_{a o a, i}$ is noise in $r_{a o a, i}$ and are defined as zero-mean gaussian distribution.

In vector form, it is represented as

$r_{\text {aoa }}=f_{\text {aoa }}(\mathrm{x})+n_{\text {aoa }}$

Where

$$
\begin{aligned}
& r_{\text {aoa }}=\left[r_{\text {aoa }, 1} r_{a o a, 2} \ldots r_{a o a, N}\right]^{T} \\
& \text { n_aoa }=\left[\mathrm{n} \_(\text {aoa,1) n_(aoa,2)...n_(aoa,N) }]^{\wedge} \mathrm{T}\right.
\end{aligned}
$$$$
f_{\text {aoa }}(x)=\left[\begin{array}{c}
\tan ^{-1}\left(\frac{y-y_{1}}{x-x_{1}}\right) \\
\tan ^{-1}\left(\frac{y-y_{2}}{x-x_{2}}\right) \\
\cdot \\
\cdot \\
\tan ^{-1}\left(\frac{y-y_{N}}{x-x_{N}}\right)
\end{array}\right]
$$

Then the source location estimation problem using AOA is to estimate $\mathrm{x}$ using $r_{a o a}$. For the development and analysis of the localization scheme, $n_{a o a, i}$ is assumed to be zero-mean and Gaussian distributed.

\section{Result analysis}

To evaluate the fixed of the proposed scheme in real world environment, a simulation experiment was carried out. To extract the training data set, a wireless sensor network of size $1500 \mathrm{~m}$ x $1500 \mathrm{~m}$ was considered. The area was partitioned into sub areas of $200 \mathrm{~m} x$ $200 \mathrm{~m}$, and each sub-area was assumed to represent a "county", able to provide 60 readings for each feature at different random locations. The area was divided into separate layers, where junctions in a single layer act as a cluster. In each layer, the nodes were assumed to be served by two systems. At each monitor, the RSS,AOA,TDOA,TOA measurements were obtained for each node and operating condition as mentioned. Two sets of 300 nodes ( 3 nodes per each county) were chosen for training and testing. The results obtained from various scenarios at which different combinations of features were considered. For each scenario the training and testing accuracy were calculated utilizing the following equation

Accuracy $=\frac{E}{N} * 100$

Where $\mathrm{E}$ is the total number of correct location estimations.

$\mathrm{N}$ is the total number of samples

Estimate of X co-ordinates

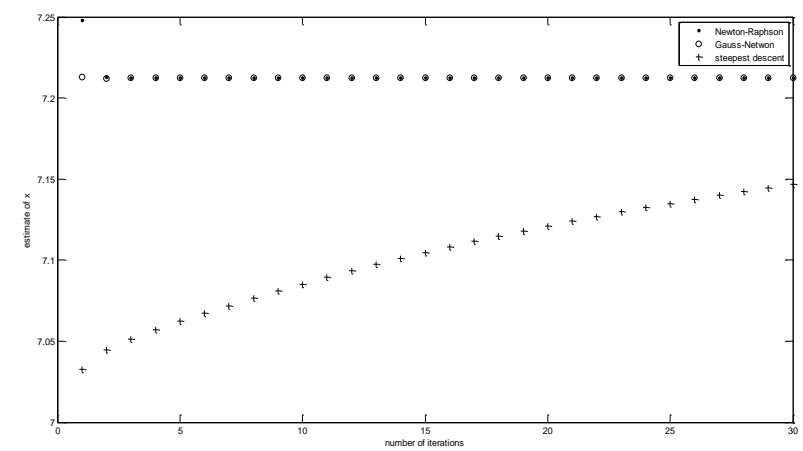

Estimate of Y co-ordinates: 


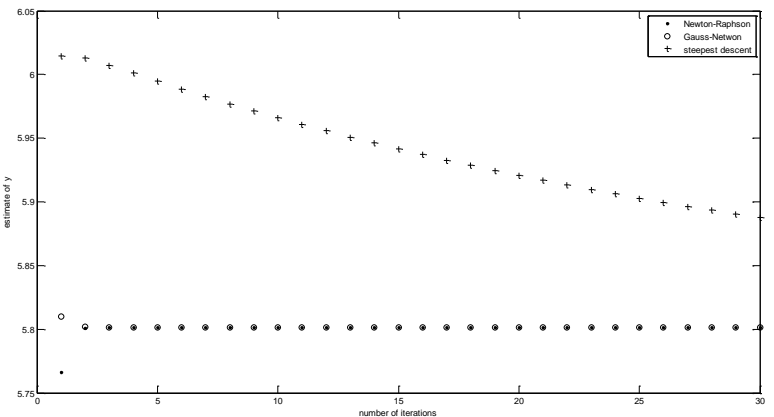

\section{Conclusion}

A new low-cost data accumulation technique for localization in WSNs was proposed. The proposed data accumulation scheme can extract different types of measurements. The process starts with known sensor node locations and sensor node readings and estimates a set of weights. These weights are used to locate unknown sensor node. In addition to low cost equipment these techniques leads to high data rates. The receiver obtained a maximum accuracy after several attempts at improvement, with roughly double the amounts of any initial accuracy readings. Furthermore, this receiver provides a proof of concept for using the proposed scheme in localization of wireless sensor networks. The proposed receiver can be best utilized in an application where an area of interest needs to obtain rather than an exact location estimation. Consider, for example, in a large corn or vegetable farm a farmer would like to locate a malfunctioning water pump. In this condition the receiver can be used to locate the area of interest (the area near the water pump). A human intervening (e.g., a farmer) then be able to use that located area of interest to determine the exact location of the pump. In such a scenario, a low cost solution to estimate an area of interest is preferable to a high cost solution that provides an exact location.

\section{References}

[1] Enyang Xu, Student Member, IEEE, Zhi Ding, Fellow, IEEE, and Soura Dasgupta, Fellow, IEEE / Source Localization in Wireless Sensor Networks From Signal Time-of-Arrival Measurements/ VOL. 59, NO. 6, JUNE 2011.

[2] Khaled Kamal Saab, Member, IEEE, and Samer Said Saab, Jr./ A Stochastic Newton-Raphson Method with Noisy Function Measurements / VOL. 23, NO. 3, MARCH 201.

[3] Maja Rosić1, Mirjana Simić1, Predrag Pejović1, Milan Bjelica1/Optimal Source Localization Problem Based on TOA Measurements/Vol. 14, No. 1, February 2017.

[4] Trung-Kien LE and Nobutaka ONO/Closed-form and Near closedform Solutions for TOA-based Joint Source and SensorLocalization/VOL.XX, NO.Y, MONTH 2016.

[5] Zohair Abu-Shaban, Student Member, IEEE, Xiangyun Zhou,Member, IEEE, and Thushara D. Abhayapala, Senior Member, IEEE./ A Novel TOA-based Mobile Localization Technique under Mixed LOS/NLOS conditions for cellular networks/ DOI 10.1109/TVT.2016.2517151 